\title{
Burrell-Optical-Kepler Survey (BOKS): A Variability Search in the Kepler Field
}

\author{
Amanda L. Proctor ${ }^{1,7}$, Steve B. Howell ${ }^{2}$, William H. Sherry ${ }^{2}$, \\ Kaspar von Braun ${ }^{3,4}$, Mark E. Everett ${ }^{5}$, John J. Feldmeir ${ }^{6}$, \\ and David R. Ciardi ${ }^{3,4}$ \\ ${ }^{1}$ Lunar and Planetary Laboratory, The University of Arizona \\ ${ }^{2}$ National Optical Astronomy Observatory \\ ${ }^{3}$ Michelson Science Center \\ ${ }^{4}$ California Institute of Technology \\ ${ }^{5}$ Planetary Science Institute \\ ${ }^{6}$ Youngstown State University \\ 7 email: aproctor@lpl.arizona.edu
}

\begin{abstract}
We present the results of eighteen non-continuous nights of time series photometric observations of a $1.25 \mathrm{deg}^{2}$ field in Cygnus centered on the NASA Kepler Mission field of view. Using the Case Western Burrell Schmidt telescope we gathered a dataset containing light curves of roughly 30,000 stars with $14<r<19$. We have statistically examined each light curve to test for variability, periodicity, and unusual light curve trends, including exoplanet transits. We present a summary of our photometric project including a characterization of the level and content of stellar variability in this field. We will also discuss our potential exoplanet candidates.
\end{abstract}

\section{Introduction}

NASA's Kepler mission will conduct a transit search in Cygnus in the hopes of finding Earth-like planets in the habitable zones of stars. The small signature of these transits makes inherent stellar variability even more of an issue. Therefore, the success of this mission depends critically on an accurate characterization of the variability in the field. To that end, the BOKS survey was designed to determine the level and type of stellar variability and the frequency of Jovian-like exoplanets in a small (1\%) part of the Kepler field.

Within our field, we find $\sim 800$ variable stars, $\sim 100$ eclipsing binaries, several hundred stars with possible rotational modulation, 100-200 candidate $\delta$ Scuti stars, two RR Lyraes, a dozen other potential pulsational variables, and a dwarf nova in outburst. The BOKS survey also resulted in three exoplanet candidates.

\section{Observations}

We were granted observing time starting on 1 September 2006 and ending on 10 October 2006. We obtained our photometric observations with the Case Western Reserve University 0.61-m, f/3.5 Schmidt telescope on Kitt Peak. The imager on the Schmidt was a SITe back-illuminated $2 \mathrm{k} \times 4 \mathrm{k}$ CCD with 15 micron $(1.5$ arcsec) pixels. We observed primarily in the SDSS $r$ band with integration times of 180 seconds and a CCD readout time of about a minute. The SITe CCD gain was fixed at 2 electrons per ADU, each pixel has a full well capacity of at least 100,000 electrons, and the read noise is 12 electrons. The typical seeing at the Schmidt is 1.2 arcsec so the PSF was sampled well enough to 
allow us to obtain photometric uncertainties of a few millimagnitudes per image for the brightest sources (see Fig. 1).

Of the 40 nights of observing, 15 were lost to weather, and an additional 7 were removed later due to bad seeing. The final data from which we will draw all samples and conclusions contains SDSS r-band light curves of about 30,000 stars with r magnitude from 14 to 19, and each light curve contains at least 1000 measurements.

\section{Light Curve Analysis}

We utilized a statistical analysis package, named OPTICSTAT, to characterize the variable nature of our light curve sample and to search for possible transits. The algorithm searches a light curve for time intervals when the mean magnitude is significantly fainter than in the preceding and following data points. To avoid detecting too many spurious light curve fluctuations as transits, we placed further constraints on the types of events reported by the transit-finding algorithm: 1) we require at least two data points during transit and at least two data points in both the pre- and post-transit light curve and 2) we search for transit durations only between one and 48 hours. Additionally, we test each light curve for periodicity, applying the Lomb method as described by Press et al. (1992). The algorithm produces a periodogram giving probabilities for sinusoidal signals in the light curves over a range of periods.

To test each light curve for general variability we attempt to fit the light curve with its mean flux and calculate the probability that the reduced $\chi^{2}$ statistic shows the data to be inconsistent with this mean flux. This test can easily fail, however, in the presence of systematic errors or even very slight uncertainties in the random magnitude errors that we assign to the data points.

\subsection{Point Source Variability}

We constrained our general variability sample to stars in the Kepler Input Catalog (KIC), which allows us to include color information about those stars. Our survey has 18,531 point sources that are common with the KIC, and thus we have additional photometric information, albeit non-contemporary, for these sources.

From this sample of $\sim 18,000$ stars, we identified $\sim 5,500(30 \%)$ that were variable. Howell (2008) discusses a well defined relation between the percentage of variable sources that will be found in any given photometric survey and that survey's photometric uncertainty. For a best precision of 4-5 mmag, as we have in the BOKS survey, the percentage of variable sources would be expected to be $\sim 36 \%$, consistent with the value actually found. Of the 5,500 variable sources, about 800 ( $15 \%$ of all the variables or $4.3 \%$ of all the stars) were found to be periodic variables. The remaining variables appear to be non-periodic.

\subsection{Periodic Variables}

We identified possible periodic stars in the BOKS data using a period probability statistic returned by OPTICSTAT. OPTICSTAT runs the phase dispersion minimization (PDM) period finding algorithm developed by Stellingwerf (1978), which returns not only a best guess period but assigns a probability to this period.

The 18,000 stars where $r<18.5$ were searched and we then added an additional criteria that the periodic amplitude had to be less than 2.5 magnitudes (amplitudes larger than this were found to be unphysical). The culled list of periodic variables was large (800) but manageable and each was phased on the best-fit period and visually inspected by eye.

We found that our sample of periodic variables had periods in the range of 0.1 to 33 days. Approximately $30 \%$ of the periodic variables have amplitudes of $>0.5$ magnitude 


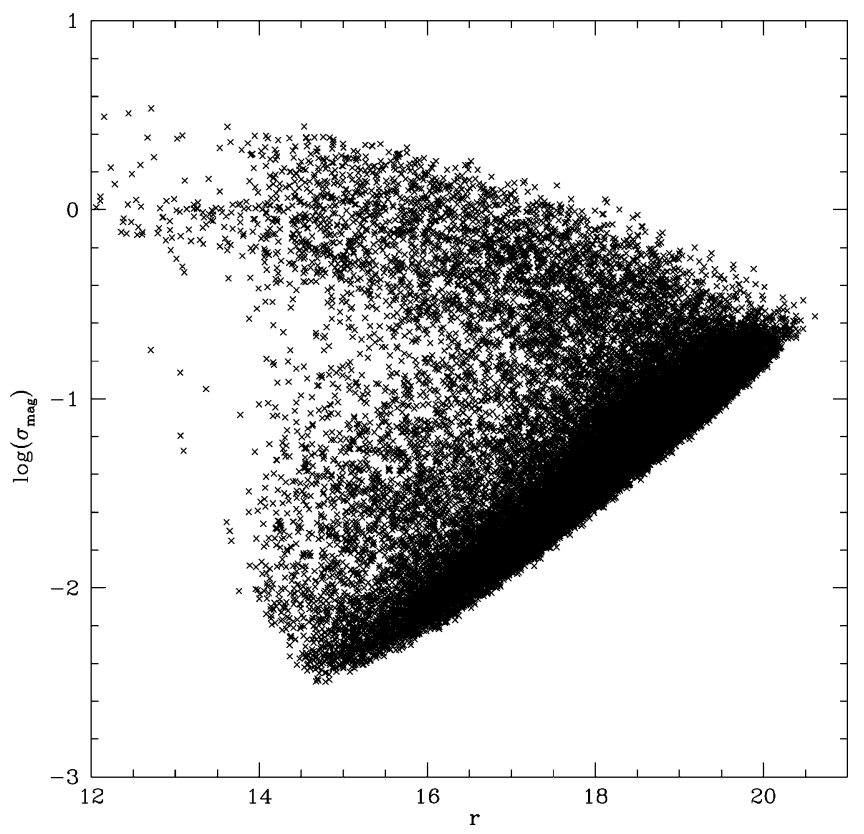

Figure 1. The standard deviation for all of the objects observed in the BOKS field as a function of $r$ magnitude. The objects brighter than $r=14$ and fainter than $r=19$ are not within the sweet spot of our observations.

while $50 \%$ have lower amplitudes (1-3\%) and periods in the 1-3 week range, indicative of rotational modulations due to star spots. Finally, $\sim 20 \%$ have amplitudes below 0.05 magnitude and periods below 2 days, consistent with pulsational variables such as $\delta$ Scuti stars. Normally, we could produce plots such as the color of the variables versus the period and use these to bolster our claims about star spot rotations (red stars) or $\delta$ Scuti variables (blue stars). However, our field lies very near the galactic plane and reddening is highly variable in both degree and location. Our attempts at sorting by color showed that most of the stars are about the same color, reddish, so only the light curves and future spectroscopy will be able to fully separate and characterize the stars.

For the large amplitude periodic variables, we find at least ten classical pulsational variables including RR Lyrae stars. We have discovered about 30 eclipsing or contact binaries, and even caught a $\mathrm{r}=20$ dwarf novae in outburst. The dwarf novae has a quiescent $r$ magnitude near 20, an outburst duration of 5.1 days, and a maximum amplitude in $r$ of 3.4 magnitudes.

\subsection{Exoplanet Transit Candidates}

After OPTICSTAT identified the most likely transit in each light curve we used the following criteria to remove candidates: 1) transit depths larger than 0.5 magnitudes and 2) events that were sharp-bottomed ( $\mathrm{V}$-shaped). We also require that the light curves show more than one transit event.

With all of the analysis possible with the data in hand complete, we are left with three planet candidates: BOKS-445069, BOKS-240959, and BOKS-252481 (shown in Fig. 2). We are currently conducting follow-up observations of these objects including multi-band photometry and spectroscopy for radial velocity measurements. 

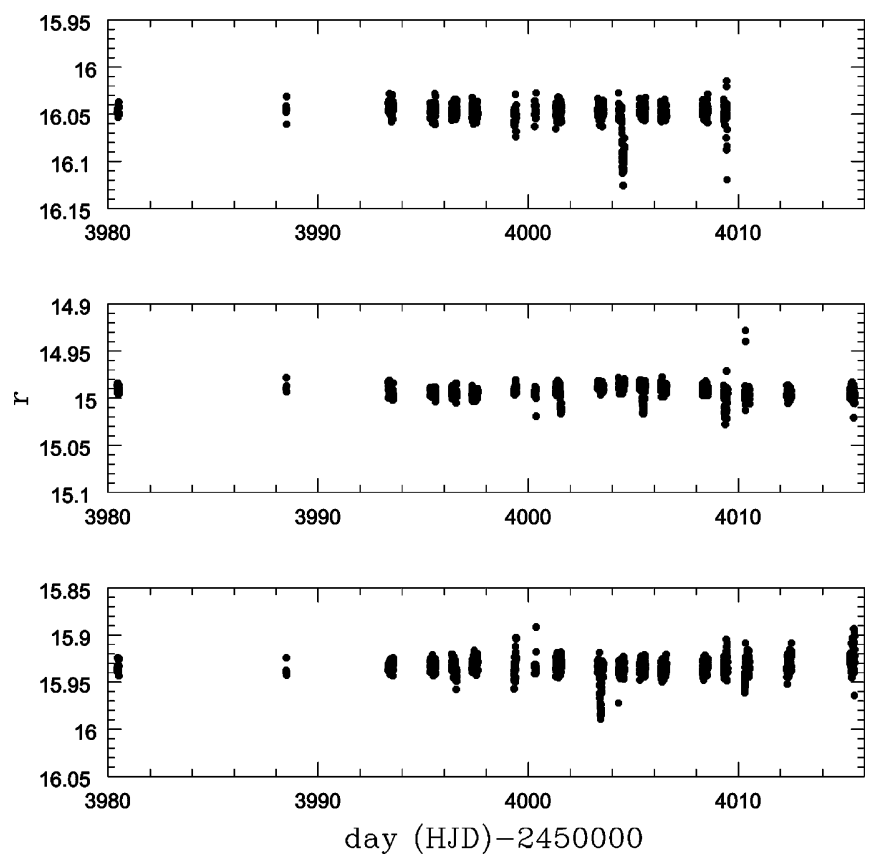

Figure 2. Light curves (in $r$ magnitudes) for our final planet candidates: BOKS-445069 (top panel), BOKS-240959 (middle panel) and BOKS-252481 (bottom panel) with typical errors of $0.012,0.014,0.012$ respectively.

\section{Conclusions}

In addition to finding three candidate transiting planets, we have identified $\sim 800$ variable stars, most of which have amplitudes of a few percent and periods of 1-3 weeks. This type of variability suggests that these are rotating low-mass dwarfs with magnetic spots. There are also low-amplitude variables with periods between 0.25 and 1.5 days. These may be $\delta$ Scuti stars. We also have $\sim 100$ eclipsing and contact binaries, as well as tens of large-amplitude pulsators such as RR Lyrae stars.

\section{References}

Howell, S. B. 2008 AN, 329, 259

Monet, D. G., Levine, S. E., Canzian, B. et al. 2003 AJ, 125, 984

Press, W. H., Teukolsky, S. A., Vetterling, W. T., \& Flannery, B. P. 1992, Num. Rec. in FORTRAN

Stellingwerf, R. F. 1978, ApJ, 224, 953 